Nanoscale force manipulation in the vicinity of a metal nanostructure

This article has been downloaded from IOPscience. Please scroll down to see the full text article.

2007 J. Phys. B: At. Mol. Opt. Phys. 40 S249

(http://iopscience.iop.org/0953-4075/40/11/S01)

View the table of contents for this issue, or go to the journal homepage for more

Download details:

IP Address: 161.111.22.141

The article was downloaded on 12/12/2012 at $11: 33$

Please note that terms and conditions apply. 


\title{
Nanoscale force manipulation in the vicinity of a metal nanostructure
}

\author{
F J García de Abajo ${ }^{1}$, T Brixner ${ }^{2}$ and W Pfeiffer ${ }^{3}$ \\ ${ }^{1}$ Instituto de Óptica, CSIC, Serrano 121, 28006 Madrid, Spain \\ 2 Physikalisches Institut, Universität Würzburg, Am Hubland, 97074 Würzburg, Germany \\ ${ }^{3}$ Fakultät für Physik, Universität Bielefeld, Universitätsstr. 25, 33516 Bielefeld, Germany \\ E-mail: jga@io.cfmac.csic.es, brixner@physik.uni-wuerzburg.de and \\ pfeiffer@physik.uni-bielefeld.de
}

Received 9 February 2007, in final form 15 April 2007

Published 16 May 2007

Online at stacks.iop.org/JPhysB/40/S249

\begin{abstract}
The tight focus of Gaussian beams is commonly used to trap dielectric particles in optical tweezers. The corresponding field distribution generates a welldefined trapping potential that is only marginally controllable on a nanometre scale. Here we investigate the influence of a metal nanostructure that is located in the vicinity of the trapping focus on the trapping potential by calculating the corresponding field and force distributions. Even for an excitation wavelength that is tuned far from the plasmonic resonance of the nanostructure, the presence of the latter alters significantly the trap potential. For the given nanostructure, a ring of spheres that is illuminated in the axial direction, a smaller focus volume is observed in comparison to free focus. The superposition of this non-resonant Gaussian field with a planar wave illumination that is tuned to the plasmonic resonance gives a handle to modify the trapping potential. Polarization and intensity of the resonant illumination allows modifying the equilibrium position of the trapping potential, thus providing means to steer dielectric particles with nanometre precision.
\end{abstract}

(Some figures in this article are in colour only in the electronic version)

\section{Introduction}

Optical tweezers allow manipulation of dielectric nanosized objects using forces as large as $100 \mathrm{pN}$ that can be controlled with about $100 \mathrm{aN}$ resolution (for recent reviews see [1-3]). The high resolution in the force measurement corresponds directly to a high spatial resolution in the $\mathrm{nm}$ range and hence allows controlling nanoscale mechanical motion. However, in a conventional optical tweezers setup the trapping potential is provided by an almost diffraction-limited focus of a Gaussian laser beam and thus it is for example not possible to 
independently control the motion of several particles that are separated by only a fraction of the wavelength. One possibility to overcome this limitation of conventional optical tweezers is the exploitation of optical near field effects that allow designing trap potentials with spatial features that are sized well below the diffraction limit. Trapping and the forces acting on nanoparticles immersed in evanescent fields or combined transversal and evanescent fields have thus been intensively investigated in theory [4-8] and experiment [9-12]. Momentum transfer and the resulting mechanical transport were demonstrated for planar evanescent fields $[9,13]$ and for a one-dimensional evanescent field distribution generated by a waveguide [14]. To achieve a highly localized trapping potential the superposition of the optical near field distribution of a sharp metal tip and a conventional trapping field has been proposed [5] and applications such as near-field photonic force microscopy become feasible [7]. Further nanoscale geometries such as surface protrusions combined with total internal reflection $[7,8,15]$, subwavelength apertures [6] and excitation of surface plasmons $[11,16]$ have been considered for the manipulation of photonic forces by evanescent fields. Recently, the surfaceplasmon-assisted manipulation of a conventional trapping potential via the superposition of an evanescent field generated by total internal reflection was experimentally demonstrated by Volpe et al [12]. The evanescent field induces forces of some $100 \mathrm{fN}$ on $0.5 \mu \mathrm{m}$ sized nanoparticles even for $500 \mathrm{~nm}$ separation between the particle and surface and an incident laser power of $18 \mathrm{~mW}$ distributed over a focal spot of $\sim 10 \mu \mathrm{m}$ in diameter [17]. The decay length for the evanescent field is about $170 \mathrm{~nm}$ and thus the related field strength of the evanescent field is already rather small. Accordingly substantial forces induced by evanescent fields are expected for suitable excitation geometries. In particular, it is conceivable that the optical near-field distribution of a nanostructure provides means to trap several particles in separate potential minima and manipulate their respective equilibrium positions independently.

Here we investigate the impact of a resonant planar wave excitation of a metal nanostructure on the trapping potential generated by non-resonant focused illumination. The nanostructure, a regular planar ring of five silver nanoparticles, is illuminated with two waves propagating in the axial direction. One wave tuned to the collective resonance of the metallic nanostructure is a planar wave, whereas the second wave is a tightly focused Gaussian-beamtuned off-resonance. In particular we emphasize the influence of the incident polarization on the trapping potential and demonstrate that the equilibrium position of dielectric nanoparticles in the vicinity of the metallic nanostructure is controllable.

\section{Methods}

We self-consistently solve the Maxwell equations for a given nanostructure and illumination conditions by means of a multiple scattering approach realized in the multiple elastic scattering of multipole expansions code (MESME) [18, 19]. This method is based on a multipole expansion of the scattered field of individual constituents of a more complex nanostructure. More precisely, the following steps are followed to solve the electromagnetic problem: (i) the external field is scattered by each of the objects that form the structure, giving rise to outgoing multipoles centred around them (single-scattering contribution), with the multipoles being defined in terms of spherical Hankel functions that reflect their outgoing-wave nature; (ii) every outgoing multipole centred around a given object is expanded in terms of propagating multipoles centred around each of the remaining objects, with the new multipoles containing spherical Bessel functions (in fact, the multipolar expansion of a light plane wave relies entirely on these functions); (iii) the latter multipoles are scattered by the object around which they are centred, giving rise to outgoing waves that add up to those obtained after first-order scattering of the incident field; (iv) this process is repeated iteratively, giving rise to the self-consistent 
relation $[18,19]$

$$
\psi_{\alpha}^{\text {ind }}=\psi_{\alpha}^{\mathrm{SS}}+t_{\alpha} \sum_{\beta \neq \alpha} H_{\alpha \beta} \psi_{\beta}^{\mathrm{ind}}
$$

where $\psi_{\alpha}^{\text {SS }}$ and $\psi_{\alpha}^{\text {ind }}$ are vectors containing the coefficients of the outgoing-wave expansion of the single-scattering contribution and the self-consistent induced field, respectively, $H_{\alpha \beta}$ is the propagation matrix that expresses an outgoing wave centred at object $\beta$ in terms of propagating spherical waves centred at object $\alpha$, and $t_{\alpha}$ is the scattering matrix of object $\alpha$ that gives the scattering outgoing-wave coefficients after interaction of propagating spherical waves with that object. The multipolar expansion is truncated at some maximum orbital angular-momentum number $l_{\max }$, so that the above equations contain $L=2 N\left(l_{\max }+1\right)^{2}$ variables, where $N$ is the number of objects $(N=5+1$ in our case) and the factor of 2 stands for the fact that we have electric and magnetic multipoles. Direct inversion of the full system of equations takes a prohibitive computation time proportional to $L^{3}$. Instead, a highly convergent iterative procedure based upon Lanzcos' method has been used to solve these equations $[18,19]$, with a computational demand proportional to $L^{2}$. We have achieved convergence after ten iterations and for $l_{\max }=15$.

The self-consistent multipolar expansion allows us to calculate the scattering cross section from the far-field scattering amplitude of the metallic structure [19]. Also, the force acting on a dielectric particle near the metallic structure is obtained from the integral of the appropriate projected components of Maxwell's stress tensor over a surface containing the particle [20], which results in a close but rather involved formula for the force in terms of the coefficients of propagating and outgoing multipoles corresponding to the moving particle alone (for more details refer to [21]).

The field and force distribution for illumination with a Gaussian beam is achieved by decomposing the Gaussian beam into 305 plane waves for each polarization component.

\section{Results and discussion}

The nanostructure chosen for the model calculation consists of $5 \mathrm{Ag}$ nanospheres with a $50 \mathrm{~nm}$ diameter arranged in a planar pentagon cluster with a $5 \mathrm{~nm}$ wide gap separating neighbouring particles (see the inset in figure 1). The total scattering cross section of the nanoparticle cluster (figure 1, solid) exhibits a strong plasmonic resonance centred at $415 \mathrm{~nm}$, slightly red shifted from the Mie resonance of non-interacting particles of the same size (figure 1, dashed). The total scattering cross section is calculated from the far-field amplitude along the forwardscattering direction, according to the optical theorem [20]. A blue-shifted mode is seen as a small side peak of this strong resonance. For longer wavelengths the scattering cross section decreases monotonically, whereas for a wavelength below $300 \mathrm{~nm}$ the interband absorption in $\mathrm{Ag}$ is dominating. A distinct minimum of the total cross section is observed at about $315 \mathrm{~nm}$. This provides a wavelength window that allows illumination of the nanostructure with minimal local field enhancement (left arrow). In contrast, excitation at the resonant wavelength of $415 \mathrm{~nm}$ (right arrow) is expected to create strong local field enhancements and thus provides means to manipulate the forces on an additional nanoparticle located in the vicinity of the $\mathrm{Ag}$ nanoparticle cluster. Here the nanostructure is immersed in a $\varepsilon=1$ environment such as air or vacuum. In the case of a calculation for a nanostructure immersed in a $\varepsilon=1.78$ medium (water), the scattering cross section is only slightly modified. The plasmon resonance is about $10 \%$ stronger and appears at a longer wavelength of $500 \mathrm{~nm}$ whereas the minimum of the response function is unaffected. Similar conclusions can be drawn when the nanostructure is 


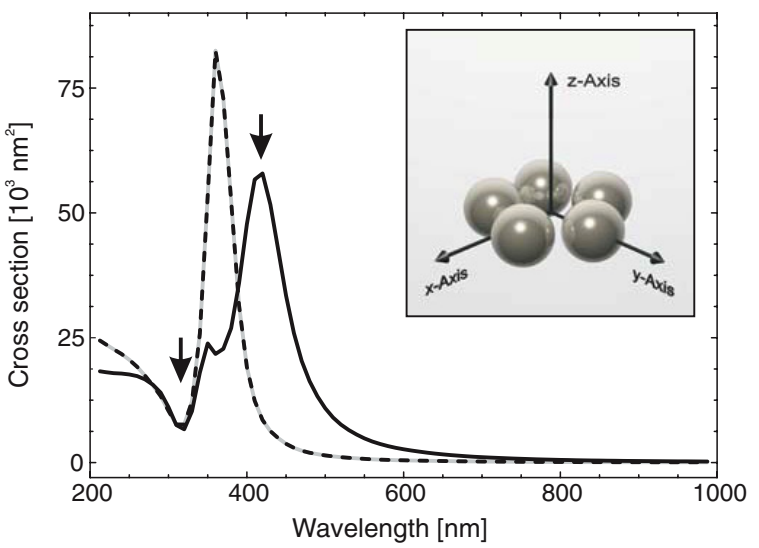

Figure 1. The total scattering cross section of the nanoparticle cluster. The geometry of the cluster, a regular planar pentagon with $5 \mathrm{~nm}$ gaps between neighbouring Ag particles $(50 \mathrm{~nm}$ diameter) is shown as inset. The incident light is unpolarized and propagating along the $z$-axis. Solid and dashed curves were obtained for interacting and non-interacting particles, respectively. The vertical arrows indicate the wavelengths for non-resonant focused and resonant planar wave illumination of the cluster.

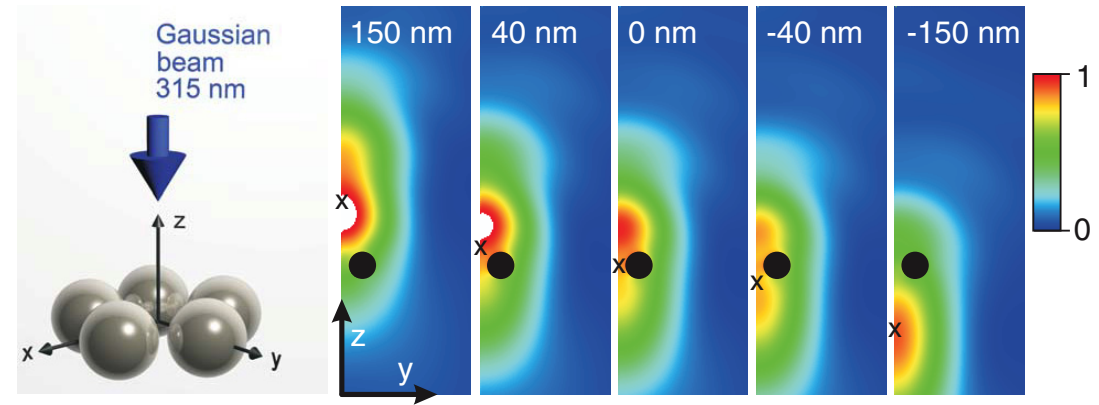

Figure 2. Field intensity $\left(|\mathbf{E}|^{2}\right)$ distribution for focused illumination at a wavelength of $315 \mathrm{~nm}$. The Gaussian beam propagates towards the negative $z$-direction. The intensities of both orthogonal polarizations are summed incoherently to represent an unpolarized beam. The horizontal (vertical) axis spans a $300 \mathrm{~nm}(900 \mathrm{~nm})$ distance in the plots. The position of the nominal focus of the Gaussian beam without cluster is indicated by crosses and explicitly mentioned for each plot. The scale goes from 0 to 1 (regions in white above that range) and the distributions are normalized to the maximum intensity at the focus in the absence of the cluster. The black circle corresponds to the cross section through an Ag nanoparticle.

deposited on a glass substrate $(\varepsilon \approx 2)$ and covered by an index matching fluid. For simplicity a surrounding medium with $\varepsilon=1$ is assumed.

As mentioned in the introduction the field control demonstrated here is based on the superposition of two different light fields: one planar wave tuned to the plasmonic resonance at $415 \mathrm{~nm}$ and an off-resonant Gaussian beam at $315 \mathrm{~nm}$. In the following the corresponding field distributions are first investigated separately. The influence of the cluster of Ag nanospheres on the field distribution of the diffraction-limited non-resonant focused Gaussian beam is shown in figure 2. Unpolarized illumination is assumed here, i.e. it is statistically averaged over all possible orientations of the incident linear polarization. Figure 2 shows cross sections of the intensity distribution for different nominal axial focus positions (marked by a cross) with 


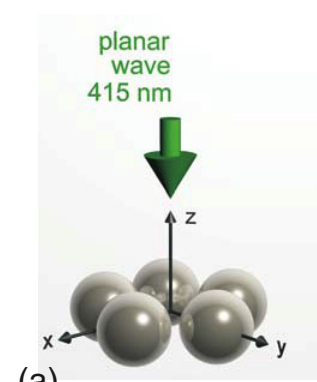

(a)
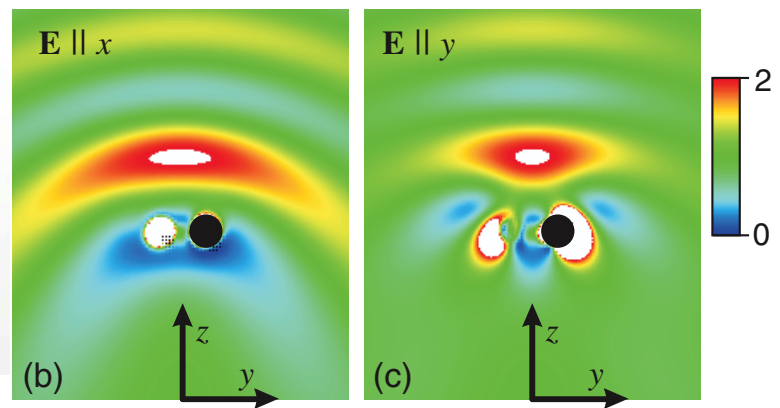

Figure 3. Field intensity $\left(|\mathbf{E}|^{2}\right)$ distribution for planar wave illumination at a wavelength of $415 \mathrm{~nm}$. (a) Excitation geometry. (b) and (c) show field intensity for incident polarization parallel to $x$ - and $y$-directions, respectively. The intensity is normalized to the intensity of the incident plane wave moving towards the negative $z$-direction. The scale goes from 0 to 2 (values above 2 in white). The horizontal (vertical) axis spans a $600 \mathrm{~nm}(690 \mathrm{~nm})$ distance in the plots. The black circle corresponds to the cross section through an Ag nanoparticle. The thin dashed lines (not shown in the online version) indicate values exceeding the shown scale.

the latter being at the centre of the focus in the absence of the nanostructure. The presence of the nanostructure significantly alters the maximum intensity as well as its axial position. For a nominal focus position above the pentagon, one observes an intensity enhancement and a reduction of the focus diameter. Both of these effects are caused by partial reflection of the incident wave leading to a standing wave pattern in front of the structure. The ring-like structure acts like a focusing mirror and thus is responsible for the stronger focusing and the resulting higher intensity in the focus. Because of the partial reflection the actual focus position is below the nominal focus for focusing at $150 \mathrm{~nm}$ and above for focusing at $40 \mathrm{~nm}$. In the case of the nominal focus position $40 \mathrm{~nm}$ above the ring a lateral focus diameter of $206 \mathrm{~nm}$ (FWHM) and an intensity enhancement of 1.05 with respect to the free focus of the Gaussian beam is achieved. The focus in this case is $93 \mathrm{~nm}$ above the structure, quite different from the nominal focus distance of $40 \mathrm{~nm}$. Even for a nominal focus position in the origin the maximum of the intensity is still localized well above the nanostructure. The intensity distribution is less altered for focus positions below the nanostructure: for a nominal focus position of $-150 \mathrm{~nm}$ the maximum intensity is reached at $-162 \mathrm{~nm}$ and the FWHM width of the distribution is $208 \mathrm{~nm}$. The longitudinal size of the focus (FWHM) for a nominal focus $40 \mathrm{~nm}$ and $150 \mathrm{~nm}$ above the nanostructure is 366 and $362 \mathrm{~nm}$, respectively, whereas the free Gaussian beam focus is extended over $420 \mathrm{~nm}$ (FWHM) for the given focusing conditions. Thus, the standing wave pattern reduces the longitudinal size of the focus by about $15 \%$, whereas the lateral size of the focus is only marginally influenced by the presence of the nanostructure. Summarizing, even for non-resonant excitation the field distribution is altered by the presence of the nanostructure and for a nominal focus position above the nanostructure a significant reduction of the longitudinal focus size is achieved. In contrast, for a focus position below the nanostructure the influence is marginal.

Now we turn to the field distribution corresponding to the resonant planar wave illumination of the nanostructure. Figure 3 shows the field intensity distribution for resonant excitation at $415 \mathrm{~nm}$ and two different polarization directions. For both polarization directions the intensity distribution is strongly altered by the presence of the nanostructure. The illuminating wave is propagating along the negative $z$-axis and thus the standing wave pattern is again located above the nanostructure leading to a maximum of the intensity at $z \approx 130 \mathrm{~nm}$. The shape of this standing wave pattern is anisotropic and varies significantly as the 

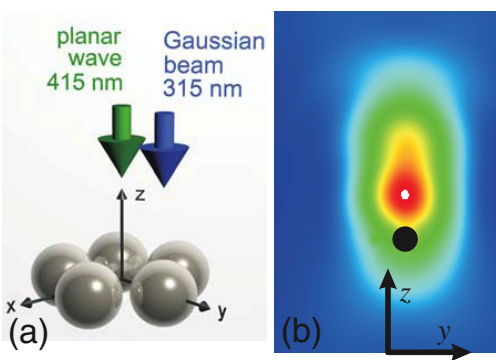
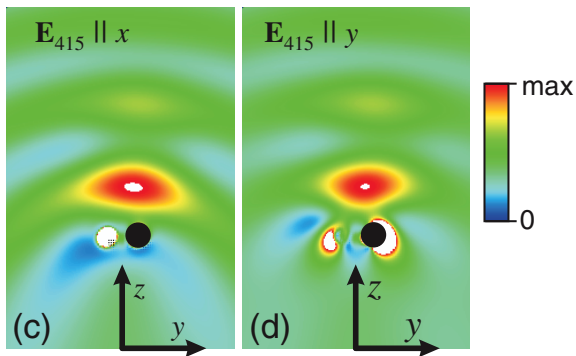

Figure 4. Field intensity $\left(|\mathbf{E}|^{2}\right)$ distribution for combined illumination. (a) Excitation geometry. The beam axis of the unpolarized Gaussian beam is displaced to the pentagon corner located on the $y$-axis. (b) Intensity distribution for illumination with the Gaussian beam at $315 \mathrm{~nm}$ with the nominal focus located $150 \mathrm{~nm}$ above the nanostructure, with the scale going from 0 to $\max =155 \mathrm{~mW} \mu \mathrm{m}^{-2}$ (values above this one in white) in units of the maximum intensity in the absence of the nanostructure. (c) and (d) show intensity distribution for combined illumination with a displaced non-resonant Gaussian beam and $200 \mathrm{~mW} \mu \mathrm{m}^{-2}$ resonant planar wave with incident polarization parallel to $x$ - and $y$-directions, respectively (the scale goes from 0 to $\max =$ $\left.550 \mathrm{~mW} \mu \mathrm{m}^{-2}\right)$. The horizontal (vertical) axis spans a $600 \mathrm{~nm}(900 \mathrm{~nm})$ distance in the plots. The black circle corresponds to the cross section through an Ag nanoparticle.

incident polarization is changed. For an incident polarization direction parallel to the $x$-axis (figure 3(b)) the region of highest intensity above the nanostructure is elongated along the $y$-direction. Below the nanostructure the illumination with a polarization parallel to the $x$-direction creates a 'shadow' in the near field intensity distribution, i.e. a reduction of the intensity with respect to the incident wave. This 'shadow' is also elongated along the $y$-axis (figure $3(\mathrm{~b})$ ). A $90^{\circ}$ rotation of the incident polarization creates a more complex field distribution with two off-axis intensity maxima below the nanostructure. The cross section shown in figure 3(c) exhibits an intensity maximum that is more strongly localized in the $y$-direction. The change of the cross section is explained by the fact that the standing wave pattern rotates with the incident polarization and that the distribution is not axially symmetric with respect to a rotation along the $z$-axis. Note that the maximum of the intensity distribution above the nanostructure, i.e. in the standing wave pattern, is always located on the $z$-axis. In contrast, the intensity distribution below the nanostructure exhibits off-axis maxima (figure 3(c)).

The goal to influence the position of the maximum intensity of the combined illumination with a non-resonant Gaussian beam and a resonant planar wave requires that at least one of the corresponding intensity distributions exhibits an off-axis maximum. To achieve this symmetry breaking, the Gaussian beam with a nominal focus of $150 \mathrm{~nm}$ above the nanostructure is laterally displaced by $47 \mathrm{~nm}$ in the $y$-direction. The beam axis is still along the $-z$-direction but now points towards the pentagon corner that coincides with the $y$-axis (figure 4(a)). First the intensity distribution corresponding to the displaced Gaussian beam is considered. This intensity distribution (figure 4(b)) has its maximum $117 \mathrm{~nm}$ above the nanostructure and $44 \mathrm{~nm}$ displaced along the $y$-axis. Hence the standing wave pattern above the nanostructure again alters the intensity distribution in the focus. The maximum is shifted towards the nanostructure and slightly displaced towards the $z$-axis with respect to the beam axis. The intensity distributions for combined illumination (resonant planar and off-resonant laterally shifted Gaussian) are shown for two orthogonal polarizations of the incident resonant planar waves in figures 4(c), (d). The superposition of the resonant and the non-resonant fields creates maxima of the resulting intensity that are clearly displaced from the $z$-axis. The position and the exact shape of the resulting intensity maximum are now controlled by the relative strength of 


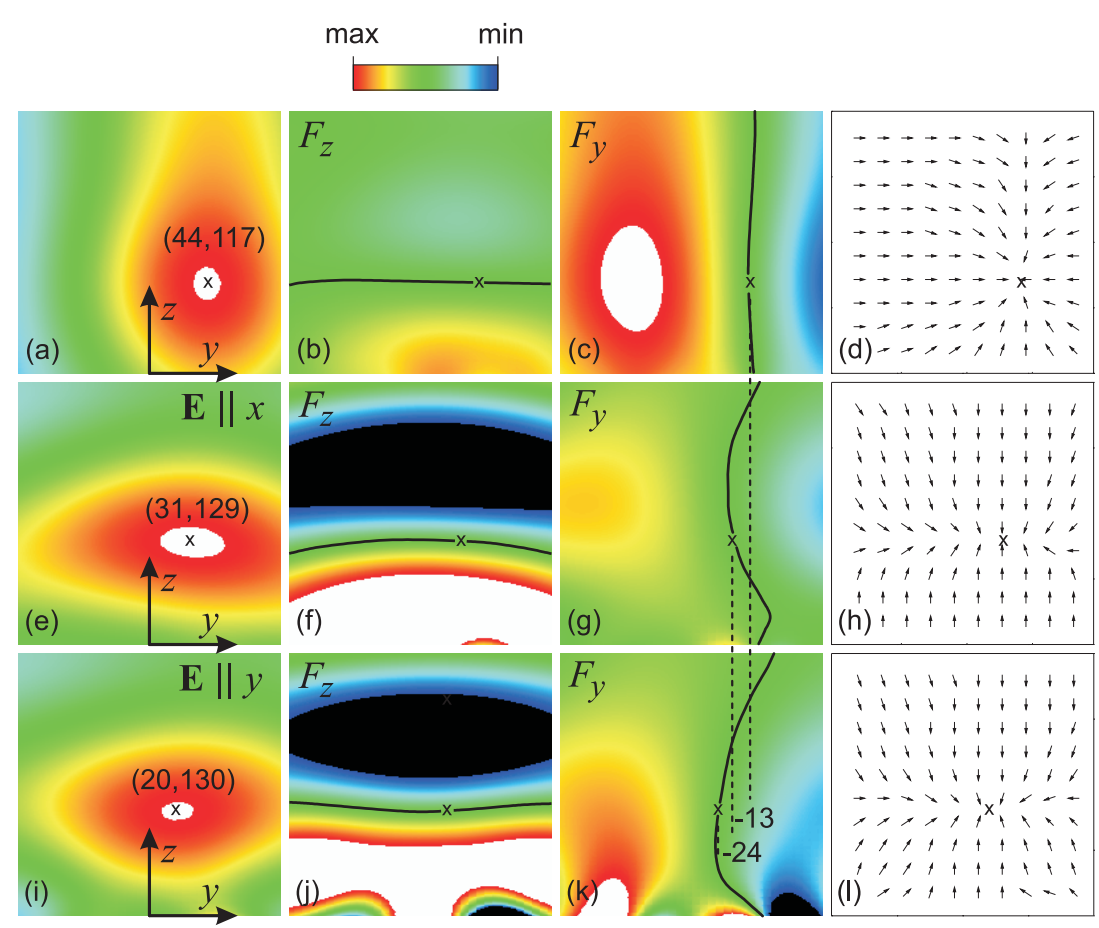

Figure 5. Field intensity (a), (e), (i) and the corresponding force distribution (b)-(d), (f), $(\mathrm{g}),(\mathrm{h}),(\mathrm{j}),(\mathrm{k}),(\mathrm{l})$ for combined illumination. Illumination with the displaced $10 \mathrm{~mW}$ Gaussian beam alone ((a)-(d)). Unpolarized Gaussian beam combined with resonant planar wave

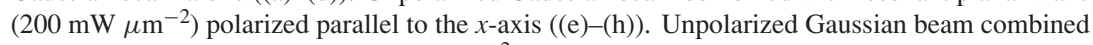
with resonant planar wave $\left(200 \mathrm{~mW} \mu \mathrm{m}^{-2}\right)$ polarized parallel to the $x$-axis $((\mathrm{i}),(\mathrm{j}),(\mathrm{k}),(\mathrm{l}))$. $200 \times 200 \mathrm{~nm}^{2}$ details of the $z-y$ plane are shown with the lower left corner corresponding to $y=$ $-100 \mathrm{~nm}$ and $z=50 \mathrm{~nm}$. The respective positions of the maximum field intensity are indicated by crosses and the vertical dashed lines give the relative displacement in $\mathrm{nm}$. The scale for the field intensity goes from 0 to $155 \mathrm{~mW} \mu \mathrm{m}^{-2}$ for (a) and reaches $550 \mathrm{~mW} \mu \mathrm{m}^{-2}$ for (e) and (i). The force scale spans -1 to $1 \mathrm{fN}$ for (b) and (c) and -3 to $3 \mathrm{fN}$ for (f), (g), (j), (k). Parts (d), (h), (l) show arrow plots for the force field distribution with an identical length scale for the arrows. The solid lines in parts (b), (c), (f), (g), (j) and (k) indicate the zero force contour. The thin dashed lines (not shown in the online version) indicate values exceeding the shown scale.

both field distributions. It is obvious that the shape and the position of the intensity maximum is modified when the resonant illumination is added, but the lateral displacement of the Gaussian focus still leads to significant asymmetry of the intensity distribution. Furthermore, the position of the maximum is influenced by the incident polarization of the planar wave (compare figure 4(c) and (d)), thus providing means to control the intensity distribution and the corresponding optical tweezers potential just by rotating the incident polarization.

Details of the intensity distribution and the corresponding force component distributions in the vicinity of the intensity maxima are shown in figure 5 for the three different illumination conditions depicted in figure 4 . The intensity maximum shifts from $y=44 \mathrm{~nm}$ to $y=31 \mathrm{~nm}$ and $y=20 \mathrm{~nm}$ as the resonant planar wave illumination is switched on with the field polarized along the $x$ - and $y$-direction, respectively. In addition the maximum moves slightly further away from the nanostructure in the latter two cases, since the resonant wavelength is longer than the non-resonant one and thus the fringe spacing of the standing wave pattern increases. With the knowledge of the field distribution it is possible to calculate the actual forces acting on a nanoparticle located in the trapping potential. The local force on a small (non-absorbing) 


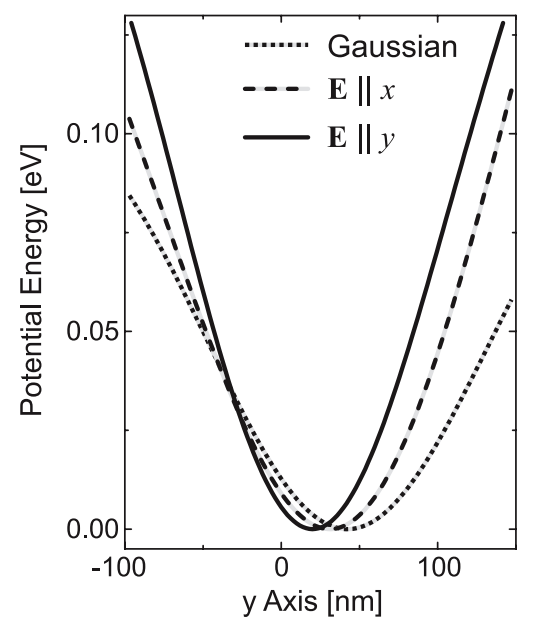

Figure 6. Potential energy for vertical displacement along the $y$-axis. The curves show cross sections through the potential minima located at $y=117 \mathrm{~nm}, y=129 \mathrm{~nm}$ and $y=130 \mathrm{~nm}$ for the Gaussian beam (dotted line) and combined illumination with polarization along the $x$ - (dashed line) and $y$-axis (solid line), respectively.

dielectric particle with polarizability $\alpha$ is given by [20]

$$
\mathbf{F}=\frac{\alpha}{2} \nabla|\mathbf{E}(\mathbf{r})|^{2}, \quad \text { with } \quad \alpha=4 \pi \varepsilon_{0} R^{3} \frac{\varepsilon-1}{\varepsilon+2},
$$

where $R$ and $\varepsilon$ are the radius of the particle and the relative dielectric constant of the particle material, respectively. An identical dielectric constant $\varepsilon=2$ is assumed for both wavelengths. The distributions of the force components $F_{z}$ and $F_{y}$ for a $R=20 \mathrm{~nm}$ particle are shown in figures $5(\mathrm{~b}),(\mathrm{f}),(\mathrm{j})$ and $5(\mathrm{c}),(\mathrm{g}),(\mathrm{k})$, respectively. The above equation for the force is in excellent agreement with direct MESME calculations including all six particles (the pentagonal structure plus the dielectric particle). The latter calculation includes the force contribution arising from scattering by the nanoparticle whereas the above given formula neglects such contribution. The quantitative agreement between both calculations shows that scattering by the dielectric particle can be neglected here. The $x$-component of the force vanishes in the $y-z$ plane because of symmetry considerations. All three force distributions provide stable trapping conditions at the location of highest field intensity (marked with $x$ in the plots). Besides the displacement of the trapping potential minimum with rotation of the incident polarization of the resonant illumination, a stronger confinement of the particle in the $z$-direction is obvious for the combined illumination (figure 5(f), (j) in comparison to (b)). Realistic incident fluences are chosen for the Gaussian beam (10 $\mathrm{mW}$ incident power corresponding to $156 \mathrm{~mW} \mu \mathrm{m}^{-2}$ peak intensity) and for the resonant planar wave illumination

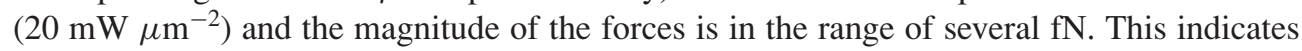
that the effects of the trapping potential modification can well be detected experimentally since the detection limit for optical tweezers lies in the range of $100 \mathrm{aN}$ [2].

Cross sections through the resulting potential energy landscape for a $20 \mathrm{~nm}$ spherical dielectric particle with $\varepsilon=2$ are shown in figure 6 . The presence of the resonant excitation significantly alters both the position of the potential energy minimum and the shape of the potential well. For the given illumination conditions the trapping energy is of the same magnitude as that measured for the effects of a purely evanescent field generated in the total reflection geometry that was investigated by Volpe et al experimentally [12]. This indicates 
that the present scheme of nanometre scale force control in the vicinity of a resonantly excited metal nanostructure is indeed feasible.

\section{Conclusion}

The results shown here demonstrate the nanoscale manipulation of the trapping potential of an optical tweezer via the polarization dependence of the scattered field of a nanostructure located in close vicinity of the trap. The maximum intensity of the combined field of a non-resonant Gaussian beam and a resonant planar wave illumination exhibits a clear spatial shift when the incident polarization of the resonant wave is changed. Thus the described illumination geometry allows controlling the position of a trapped nanoparticle without any mechanical displacements involved. To achieve this control over the trap position in space some symmetry breaking is required. Here this is achieved by displacing the Gaussian beam with respect to the symmetry axis of the nanostructure. However, it is conceivable to use an asymmetric nanostructure to achieve a similar effect, making the proposed scheme rather flexible. To our knowledge the superposition of a resonant plasmonic field with a non-resonant trapping potential represents a new concept to control optical tweezers on the nanoscale. The concept presented here differs from other proposed schemes, such as force distributions in the vicinity of nanoholes [6] or microscopic tips [22], since it provides controllability of a well-defined trap potential that is well separated from the nanostructure. In the case of the nanoholes the variation of the force distribution with varying incident polarizations has been investigated [6], but the purely evanescent character of the involved fields generates a situation where the highest intensities appear on the surface of the nanostructure and thus the resulting forces lead to the attachment of the trapped particles to the nanostructure. In contrast, the scheme presented here exploits the resonantly scattered field of the nanostructure, whose influence depends on the incident polarization, thus allowing one to control the trap potential. In the present demonstration the control of the trap position is rather limited. However, we expect that a suitable choice of other nanostructures will provide an even higher degree of spatial control. In particular it is conceivable to design nanostructures that produce scattered field distributions supporting two minima of the trapping potential, which can then also be controlled by the incident polarization, allowing one for example to manipulate the separation of two trapped nanoparticles at distances well below the wavelength. Recent theoretical [23] and experimental works [24] demonstrate control of the field excitation in the vicinity of a metal nanostructure via polarization pulse shaping. Hence, we expect that the use of a polarization-shaped laser could provide an even better controllability of the trapping potential and might in future allow dynamic mechanical control of light particles such as atoms or molecules.

\section{Acknowledgment}

TB thanks the DFG for an Emmy Noether Fellowship.

\section{References}

[1] Ashkin A 1997 Proc. Natl Acad. Sci. USA $944853-60$

[2] Grier D G 2003 Nature 424 810-6

[3] Neuman K C and Block S M 2004 Rev. Sci. Instrum. 75 2787-809

[4] Jhe W, Ohtsu M, Hori H and Friberg S R 1994 Japan J. Appl. Phys. Lett. 33 L1680-2

[5] Novotny L, Bian R X and Xie X S 1997 Phys. Rev. Lett. 79 645-8 
[6] Okamoto K and Kawata S 1999 Phys. Rev. Lett. 83 4534-7

[7] Nieto-Vesperinas M, Chaumet P C and Rahmani A 2004 Phil. Trans. R. Soc. A $362719-37$

[8] Quidant R, Petrov D and Badenes G 2005 Opt. Lett. 30 1009-11

[9] Kawata S and Sugiura T 1992 Opt. Lett. 17 772-4

[10] Sasaki K, Hotta J, Wada K and Masuhara H 2000 Opt. Lett. 25 1385-7

[11] Gu M, Haumonte J B, Micheau Y, Chon J W M and Gan X 2004 Appl. Phys. Lett. 84 4236-8

[12] Volpe G, Quidant R, Badenes G and Petrov D 2006 Phys. Rev. Lett. 96238101

[13] Almaas E and Brevik I 1995 J. Opt. Soc. Am. B 12 2429-38

[14] Kawata S and Tani T 1996 Opt. Lett. 21 1768-70

[15] Chaumet P C, Rahmani A and Nieto-Vesperinas M 2002 Phys. Rev. Lett. 88123601

[16] Garcés-Chávez V, Dholakia K and Spalding G C 2005 Appl. Phys. Lett. 86031106

[17] Quidant R 2007 Personal communication

[18] García de Abajo F J 1999 Phys. Rev. Lett. 82 2776-9

[19] García de Abajo F J 1999 Phys. Rev. B 60 6086-102

[20] Jackson J D 1975 Classical Electrodynamics (New York: Wiley)

[21] García de Abajo F J 2004 Phys. Rev. B 70115422

[22] Novotny L, Hecht B and Pohl D W 1997 J. Appl. Phys. 81 1798-806

[23] Brixner T, García de Abajo F J, Schneider J and Pfeiffer W 2005 Phys. Rev. Lett. 95093901

[24] Aeschlimann M, Bauer M, Bayer D, Brixner T, García de Abajo F J, Pfeiffer W, Rohmer M, Spindler C and Steeb F 2007 Nature 446 301-4 